\title{
Characteristics of Pyrolytic Graphite as a Neutron Monochromator
}

\author{
M. Adib ${ }^{1}$, N. Habib ${ }^{1}$, M. S. El-Mesiry ${ }^{1} \&$ M. Fathallah ${ }^{2}$ \\ ${ }^{1}$ Reactor Physics Department, NRC, AEAE, Cairo, Egypt \\ ${ }^{2}$ Faculty of Science, Jazan University, KSA \\ Correspondence: M. Adib, Reactor Physics Department, NRC, AEAE, Cairo, Egypt. E-mail: \\ mamdouhshihata@yahoo.com
}

Received: February 14, 2012 Accepted: February 27, 2012 Online Published: May 3, 2012

doi:10.5539/eer.v2n1p35 URL: http://dx.doi.org/10.5539/eer.v2n1p35

\begin{abstract}
Pyrolytic graphite (PG) has become nearly indispensable in neutron spectroscopy. Since the integrated reflectivity of the monochromatic neutrons from PG crystals cut along its c-axis is high within a wavelength band from $0.1 \mathrm{~nm}$ up to $0.65 \mathrm{~nm}$. The monochromatic features of PG crystal is detailed in terms of the optimum mosaic spread, crystal thickness and reactor moderating temperature for efficient integrated neutron reflectivity within the wavelength band. A computer code Mono-PG has been developed to carry out the required calculations for the PG hexagonal close-packed structure.

Calculation shows that, $2 \mathrm{~mm}$ thick of PG crystal having $0.3^{0}$ FWHM on mosaic spread are the optimum parameters of PG crystal as a monochromator at selected neutron wavelength shorter than $0.2 \mathrm{~nm}$. However, the integrated neutron intensity of $2^{\text {nd }}$ and $3^{\text {rd }}$ orders from a thermal reactor flux is even higher than that of the $1^{\text {st }}$ order one at neutron wavelengths longer than $0.2 \mathrm{~nm}$. While, from a cold reactor flux, the integrated neutron intensity of the $1^{\text {st }}$ order within the wavelength band from 0.25 up to $0.5 \mathrm{~nm}$ is higher than the $2^{\text {nd }}$ and $3^{\text {rd }}$ ones.
\end{abstract}

Keywords: thermal neutron monochromators, pyrolytic graphite crystal

\section{Introduction}

A monochromator is a single-crystal that selects the neutron wavelength according to Bragg's law $n \lambda=2 d \sin \theta$. The range of wavelengths accepted depends on the crystal structure and its mosaicity. A larger mosaicity increases the number of monchromatic neutrons that will make it to the sample, while reduces its wavelength resolution (Lovesey, 1987).

Common materials used as monochromator crystals are pyrolytic Graphite (PG), Silicon, Copper, Beryllium, Iron, and Heusler crystals for use in polarized neutron scattering experiments. The choice of monochromator depends on the range of incident energies required for the experiment and the desired energy resolution.

However, a beam of monochromatic neutrons, selected from the spectrum of a nuclear reactor by means of diffraction by a monochromator crystal, will in general contaminate with higher-order components. Consequently the use of a filter is indispensable to eliminate higher order contaminations (Arzi, 2001).Therefore, silicon (111) reflection comes with the added advantage that second order $(n=2)$ neutrons are forbidden (Mitchell, 2006).

Now-a-days PG is commonly used as high efficient neutron monochromator and filter since it has low absorption cross section and high scattering one (Adib et al., 2011; Adib et al., 2006a; Adib et al., 2006b; Mildner et al., 2001; Mook et al., 2001). Moreover now a bulk PG crystal with small mosaic spread at reasonable price is available (Ohler et al., 1997).

Therefore in the present work, a feasibility study of using PG as a monochromator is detailed in terms of optimum parameters of its mosaic spread and thickness taking into consideration the ratio of higher order reflections to the first one.

\section{Theoretical Treatment}

The main parameters determining the quality of PG crystal as a neutron monochromator are: the wavelength band of the reflected monochromatic neutrons, reflecting power $\mathbf{P}_{h k l}^{\theta}$, wavelength spread of the reflected beam and the ratio of higher order contaminations to the first one. 
In pyrolytic graphite the crystallites are aligned to a high degree with their hexagonal c-axes parallel, whereas the a-axes are oriented at random. Therefore, the reflected neutrons are from the $(00 l)$ planes satisfying the Bragg equation (Bacon, 1975).

$$
\mathrm{n} \lambda=2 \mathrm{~d}_{00 l} \sin \theta_{00 l}
$$

Where, $n$ is the order of reflection, $\theta_{00 l}$ is the glancing angle to the $(00 l)$ plane.

In case of PG crystal the reflecting power is due to reflection from (002) plane and its higher orders, where the PG crystal is cut perpendicular to its c-axis. Thus the reflected power of the (002) plane at glancing angle $\theta$ within $\mathrm{d} \theta$ is given by (Bacon, 1975):

$$
P_{00 l}^{\theta} d \theta=\frac{a d \theta}{1+a+(1+2 a)^{1 / 2} \operatorname{coth}\left[A(1+2 a)^{1 / 2}\right]}
$$

Where

$$
\begin{gathered}
A=\mu t_{o} / \gamma_{o} \\
a=\frac{Q_{00 l}}{\mu} W(\theta)
\end{gathered}
$$

in which $\mu$ is the linear absorption coefficient, $\mathrm{Q}_{001}$ the well-known crystal-graphic quantity, $\mathrm{t}_{0}$ the PG thickness and $\gamma_{o}$ the direction cosine of the incident neutron beam relative to the inward normal the crystal face. $W(\theta)$ has a Gaussian distribution with standard deviation $\eta$ on mosaic blocks of single crystal. The integrated reflectively $R^{\theta}$ is given by:

$$
R^{\theta}=\int_{-\infty}^{+\infty} P_{00 l}^{\theta} d \theta
$$

While the reflected intensity $I_{\operatorname{Re} f}$ from PG crystal when a neutron beam having Maxiwellain distribution $\Phi(\lambda)$ is given by:

$$
I_{\operatorname{Re} f}=\Phi(\lambda) * P_{00 l}^{\theta}
$$

Where $\Phi(\lambda)$ having neutron gas temperature $T$ is given by (Gurevich, \& Tarasov, 1968):

$$
\phi(\lambda)=\frac{\text { constant }}{\lambda^{5}} \exp \left(-h^{2} / 2 m k T \lambda^{2}\right)
$$

A computer code Mono-PG has been developed to carry out the calculation of distribution of $\mathbf{P}_{00 l}^{\theta}$ and of the reflected intensity $\mathrm{I}_{\text {Ref }}$ as a function of neutron wavelength, mosaic spread, setting glancing angle, thickness of PG crystal and reactor moderating temperature T. The Mono-PG code is an adapted version of the computer package GRAPHITE written by (Adib \& Fathalla, 2007). The flowchart of the computer code Mono-PG is displayed in Figure 1. 


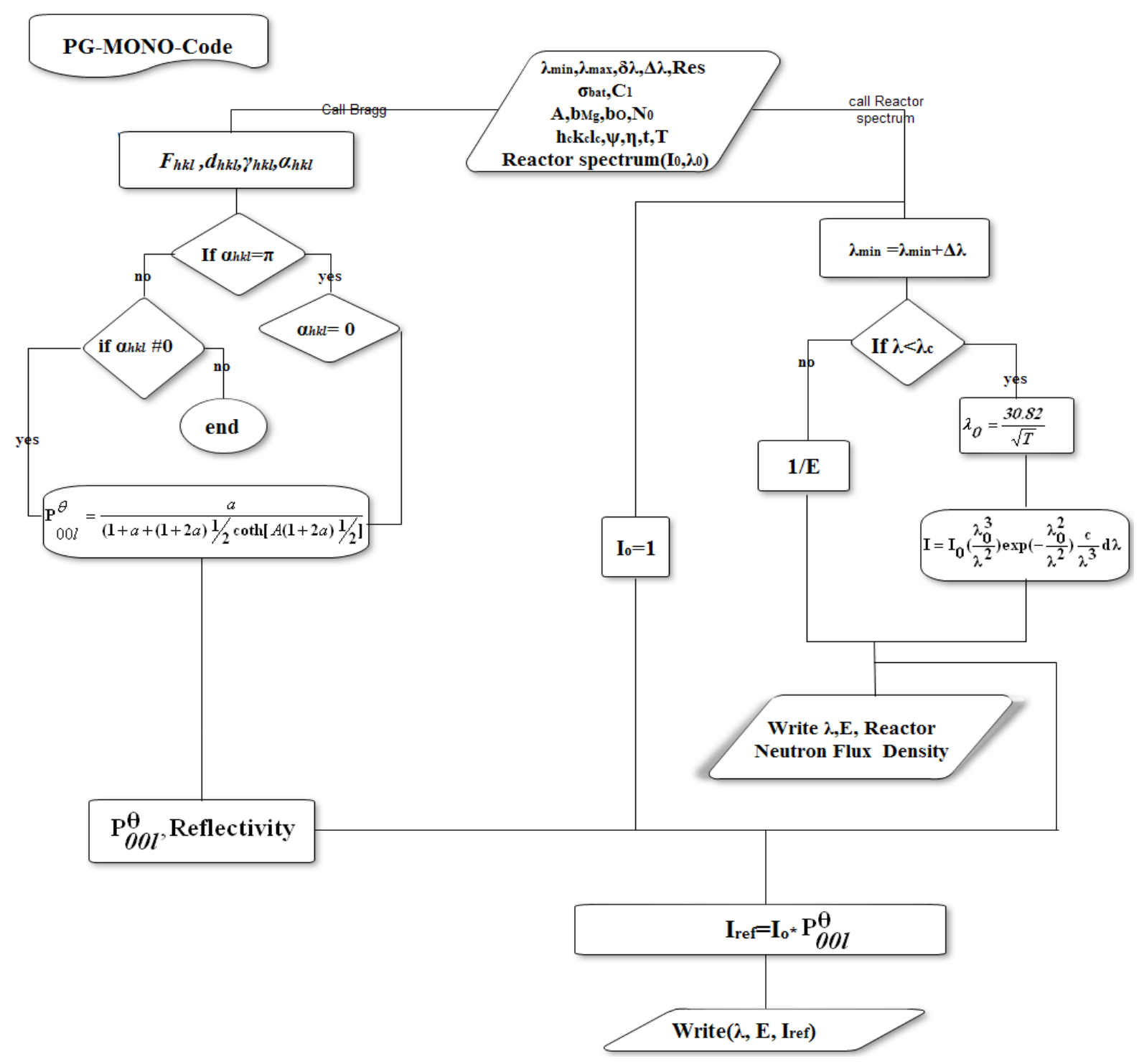

Figure 1. The flowchart of PG-Mono code

\section{Monochromatic Features of PG Crystal}

The distribution of the reflected neutrons $\mathbf{P}_{002}^{\theta}$ from $\mathrm{PG}$ crystal cut along c-axis, as a function of mosaic spread were calculated, assuming the following input parameters: PG thickness $t=2 \mathrm{~mm}$ set at glancing angle $\theta=23.45^{\circ}$, FWHM of incident beam divergence is $0.4^{\circ}$ and the wavelength band was from $\lambda_{\min }=0.001 \mathrm{~nm}$ to $\lambda_{\max }=0.4 \mathrm{~nm}$ with $\Delta \lambda=0.0005 \mathrm{~nm}$. The result of calculation is displayed in Figure 2 . 


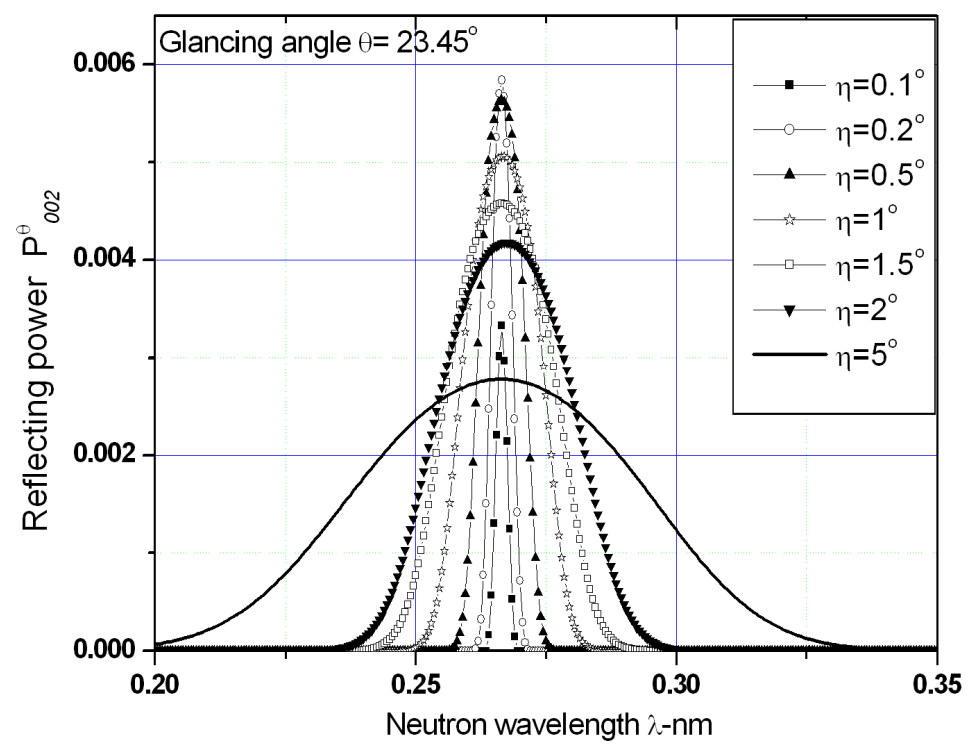

Figure 2. The wavelength distribution of the reflectivity $\mathbf{P}_{002}^{\theta}$ from PG at various $\eta$

From the figure, the maximum of reflectivity $P_{002}^{\max }$ was at $\lambda_{002}=0.269 \mathrm{~nm}$ which was found independent of PG mosaic spread $\eta$ and in agreement with that calculated from Bragg equation at glancing angle $\theta=23.45^{\circ}$ The wavelength resolution $(\Delta \lambda / \lambda)$ as a function of mosaic spread was determined as a ratio of the FWHM of the distribution $\mathbf{P}_{002}^{\theta}$ to the wavelength $\lambda_{002}$. The values of maximum reflectivity $P_{002}^{\max }$ and $\Delta \lambda / \lambda$ for versus $\eta$ are displayed in Figure 3. Figure 3 shows that $\Delta \lambda / \lambda$ is increasing with the increase of the mosaic spread $\eta$. This is consistent of the fact that low quality crystal (i.e. high value of $\eta$ ) the distribution of the reflected neutrons is broadened consequently the resolution becomes worst.

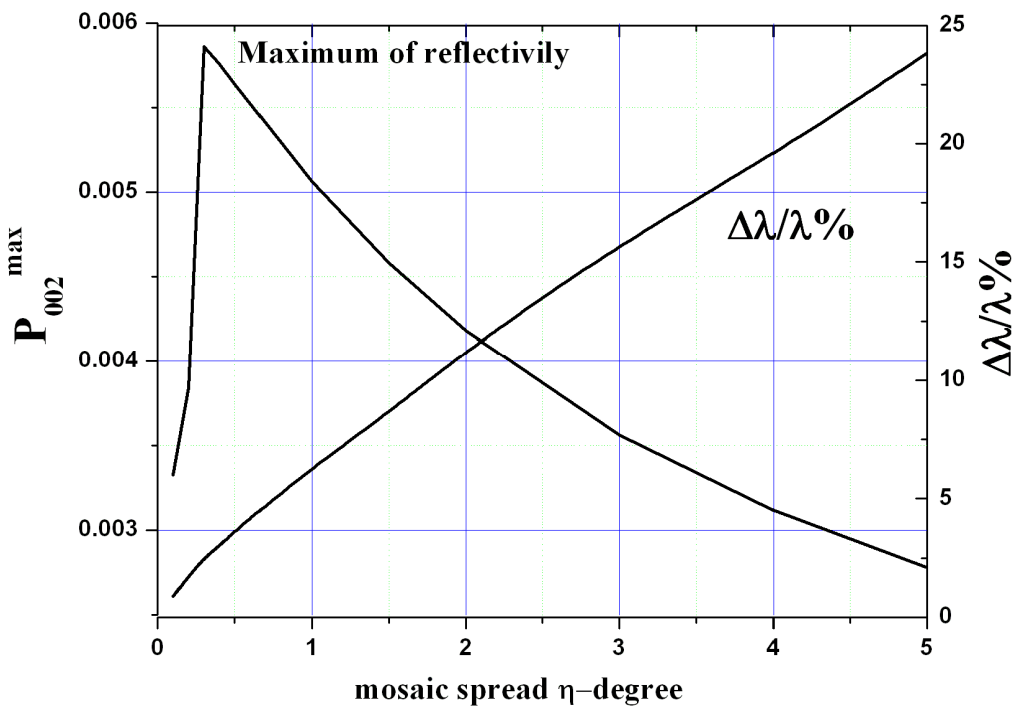

Figure 3. Maximum $\mathbf{P}_{002}^{\theta}$ and $\Delta \lambda / \lambda$ versus $\eta$ 
Figure 3 shows also that $P_{002}^{\max }$ reaches a maximum value at FWHM on mosaic spread $\eta=0.3^{0}$. Such behavior may be due to the fact that the assumed incident neutron beam divergence $\left(0.4^{0}\right)$ is comparable with the mosaic spread value. Therefore the optimum FWHM on mosaic spread $\eta$ of PG when it used as a monochromator is $0.3^{0}$ at high resolution of the reflected beam $(\Delta \lambda / \lambda \prec 3 \%)$.

The integrated reflectivity $\mathrm{R}^{\theta}(\mathrm{n})$ of the monochromatic neutrons of $1^{\text {st }}$ order reflection from $2 \mathrm{~mm}$ thick PG $(\eta=$ $0.3^{0}$ ) crystal along with the higher order contaminations accompanying the $1^{\text {st }}$-one are calculated versus the glancing angle $\theta$ i.e. versus neutron wavelength $\lambda_{002}$. The result of calculation is displayed in Figure 4. It would appear that $2 \mathrm{~mm}$ thick PG $\left(0.3^{0}\right)$ is a good neutron monochromator in a wide wavelength band from $0.1 \mathrm{~nm}$ up to $0.65 \mathrm{~nm}$.

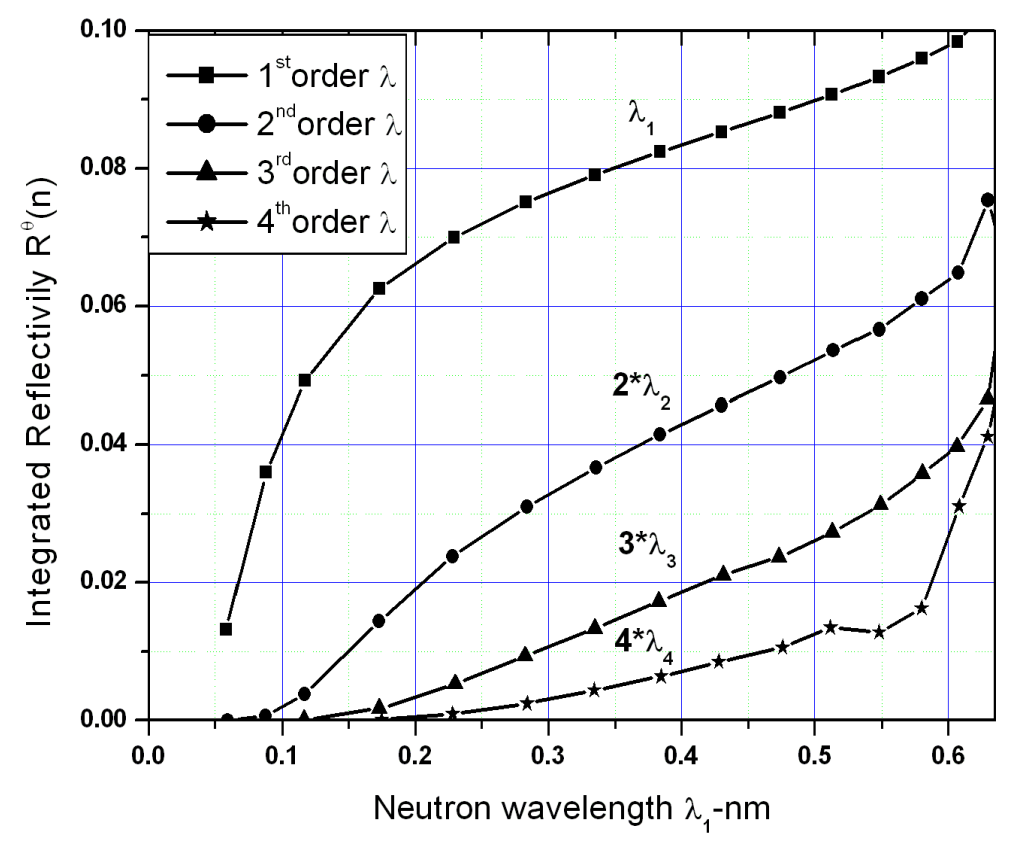

Figure 4 . The integrated reflectivity $\mathrm{R}^{\theta}(\mathrm{n})$ versus neutron wavelength $\lambda_{002}$

To show the effect of higher order contaminations accompanying the first one, the reflected neutrons from $2 \mathrm{~mm}$ thick PG (FWHM on mosaic spread $=0.3^{\circ}$ ) set at glancing angle $\theta=23.45^{\circ}$ was calculated. The result of calculation is displayed in Figure 5. It is apparent that, the integrated reflectivity of the second-order reflection is less than that of first-order one and also the integrated of higher ones are even more less. This is in agreement with the Laue behavior of Bragg scattering (Lovesey, 1987).

The integrated reflectively $R^{\theta}(1)$ from PG $\left(0.5^{\circ}\right.$ on mosaic spread $)$ crystal set at glancing angle $\theta=23.45^{\circ}$ was calculated versus PG thickness. The result of calculation is listed in Table 1. For comparison, the contamination factors $R(n)$ of higher orders versus thickness $\mathrm{t}_{0}$ are calculated as $R(n)=R^{\theta}(1) / R^{\theta}(n)$, where $n=2,3,4$ and the result is also listed in Table 1. One can notice that, contamination $R(n)$ is increasing more rapidly than the integrated reflectivity $R^{\theta}(1)$ with increasing $\mathrm{t}_{0}$. Consequently the thinner the PG thickness is the better, however one must take into account the value of effect-to-back ground of a thin PG with low integrated reflectivity of $1^{s t}-$ order . 


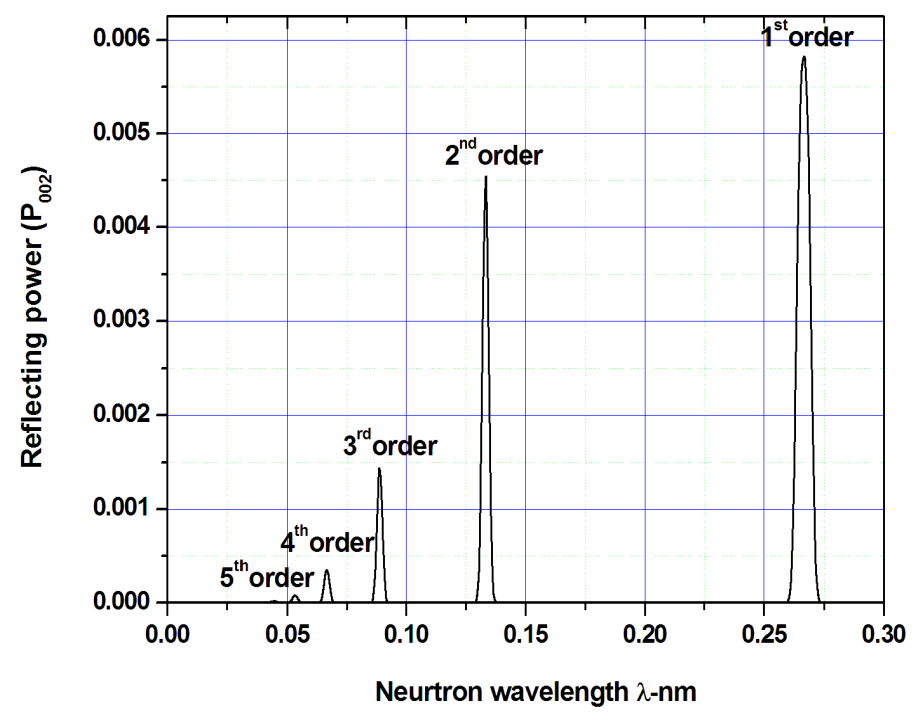

Figure 5. The $1^{\text {st }}$ and higher orders reflecting power $\mathbf{P}_{002}^{\theta}$ distribution

Table 1. $R^{\theta}(1) \& R(n)$ versus PG thickness $\mathrm{t}_{\mathrm{o}}$

\begin{tabular}{ccccc}
\hline $\begin{array}{c}\text { Thickness } \\
\mathrm{t}_{\mathrm{o}}(\mathrm{mm})\end{array}$ & $R^{\theta}(1)$ & $R(2)$ & $R(3)$ & $R(4)$ \\
\hline 0.2 & 0.036 & 0.133 & 0.025 & 0.006 \\
0.5 & 0.063 & 0.179 & 0.036 & 0.008 \\
0.8 & 0.078 & 0.217 & 0.046 & 0.010 \\
1.0 & 0.085 & 0.239 & 0.052 & 0.012 \\
2.0 & 0.108 & 0.319 & 0.079 & 0.018 \\
5.0 & 0.134 & 0.452 & 0.142 & 0.036 \\
8.0 & 0.146 & 0.519 & 0.189 & 0.051 \\
10.0 & 0.152 & 0.547 & 0.214 & 0.060 \\
12.0 & 0.156 & 0.571 & 0.237 & 0.069 \\
15.0 & 0.161 & 0.598 & 0.266 & 0.082 \\
18.0 & 0.165 & 0.618 & 0.291 & 0.094 \\
20.0 & 0.168 & 0.625 & 0.305 & 0.101 \\
25.0 & 0.172 & 0.651 & 0.337 & 0.137 \\
\hline
\end{tabular}

One can conclude that PG crystal with thickness from 2 to $5 \mathrm{~mm}$ is preferable thickness, for PG crystal when it used as a neutron monochromator. From Table 1 one can also notice that for $2 \mathrm{~mm}$ thick the contaminations of $2^{\text {nd }}$ and $3^{\text {rd }}$ orders are $32 \%$ and $7.9 \%$ respectively. These contaminations are true when the incident neutron beam distribution is constant. However the incident neutron beam distribution from steady state reactor obeys Maxiwellain distribution with neutron gas temperature $\mathrm{T}$.

Therefore the neutron intensities reflected from PG were calculated at both room 300K (thermal reactor flux) and liquid Hydrogen temperatures (cold reactor flux) of the reactor moderator. The result of calculation is displayed in Figure $6 \mathrm{a} \& 6 \mathrm{~b}$. From Figure $6 \mathrm{a}$, one can notice that the integrated reflected neutron intensity of $2^{\text {nd }}$ and $3^{\text {rd }}$ orders from thermal reactor flux are even higher than that from the $1^{\text {st }}$ order one. So the use of PG crystal as an efficient neutron monochromator is limited. To improve such case a neutron filter is essential. While, Figure $6 \mathrm{~b}$ shows that, the intensities of higher order reflections from cold reactor flux are too small with respect to the $1^{\text {st }}$ order one. So, 
for the neutron flux which exit from a channel of a steady state reactor with neutron gas temperature close to hydrogen one, if available, there is no need to use a neutron filter.
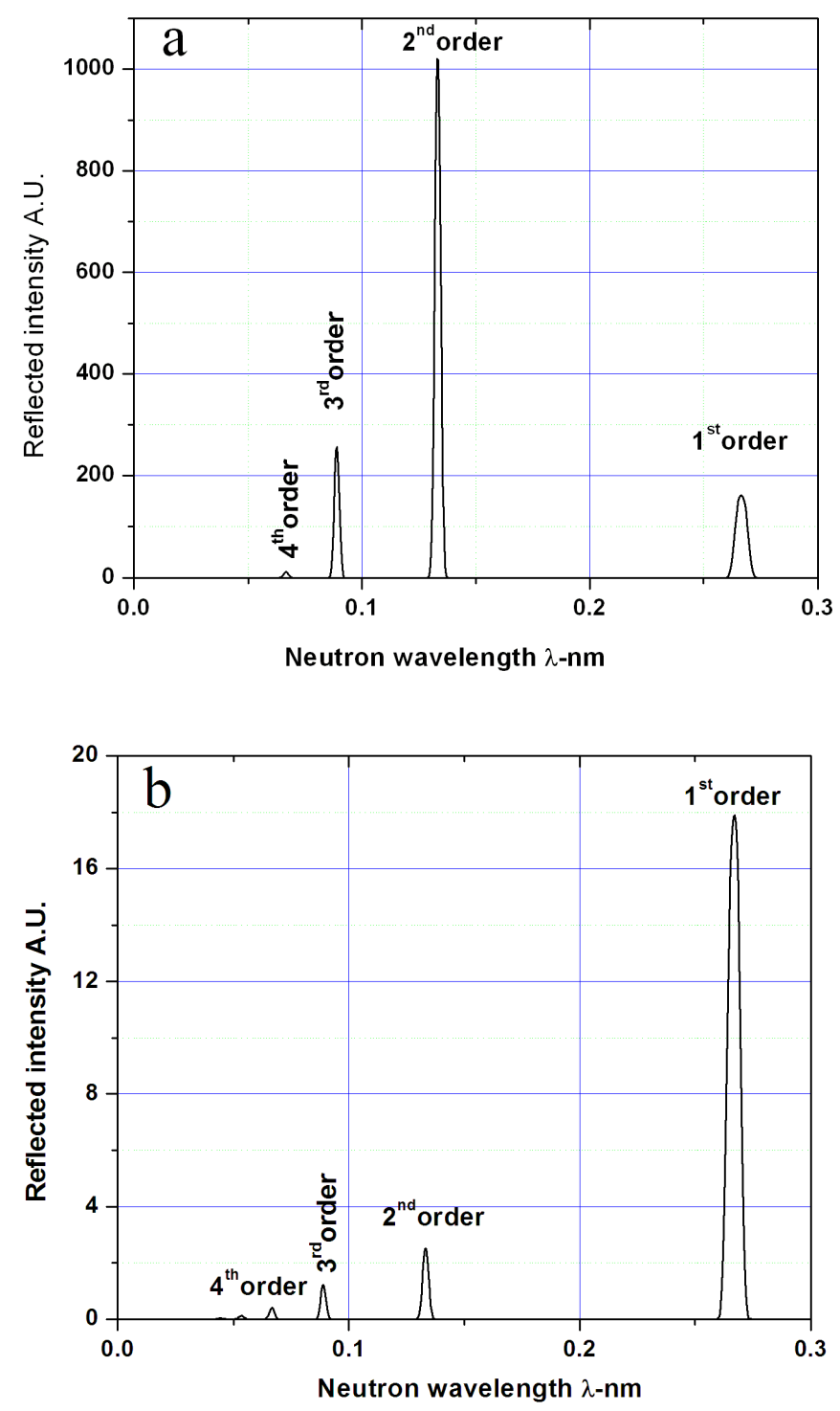

Figure 6. The neutron intensities reflected from PG monochromator

Since the reflected neutron monochromatic intensities and its higher orders contaminations from PG crystal was found to depend upon both the reactor moderating temperature and the value of the selected monochromatic wavelength, therefore the integrated neutron monochromatic intensities $I_{\operatorname{Re} f}$ reflected from $2 \mathrm{~mm}$ thick PG (FWHM on mosaic spread $=0.3^{0}$ ) were calculated as a function of the neutron wavelength, i.e. glancing angle $\theta$. The result of calculation is displayed in Figure 7a for incident thermal reactor neutron beam, while for cold reactor one in Figure 7b. Figure 7a shows that, for incident thermal reactor beam, the reflected monochromatic neutrons of $1^{\text {st }}$-order, within the wavelength band from $0.08 \mathrm{~nm}$ up to $0.15 \mathrm{~nm}$ is accompanying with less than $5 \%$ of higher order contaminations. Therefore, one can use PG as a thermal neutron monochromator without need for a filter. 

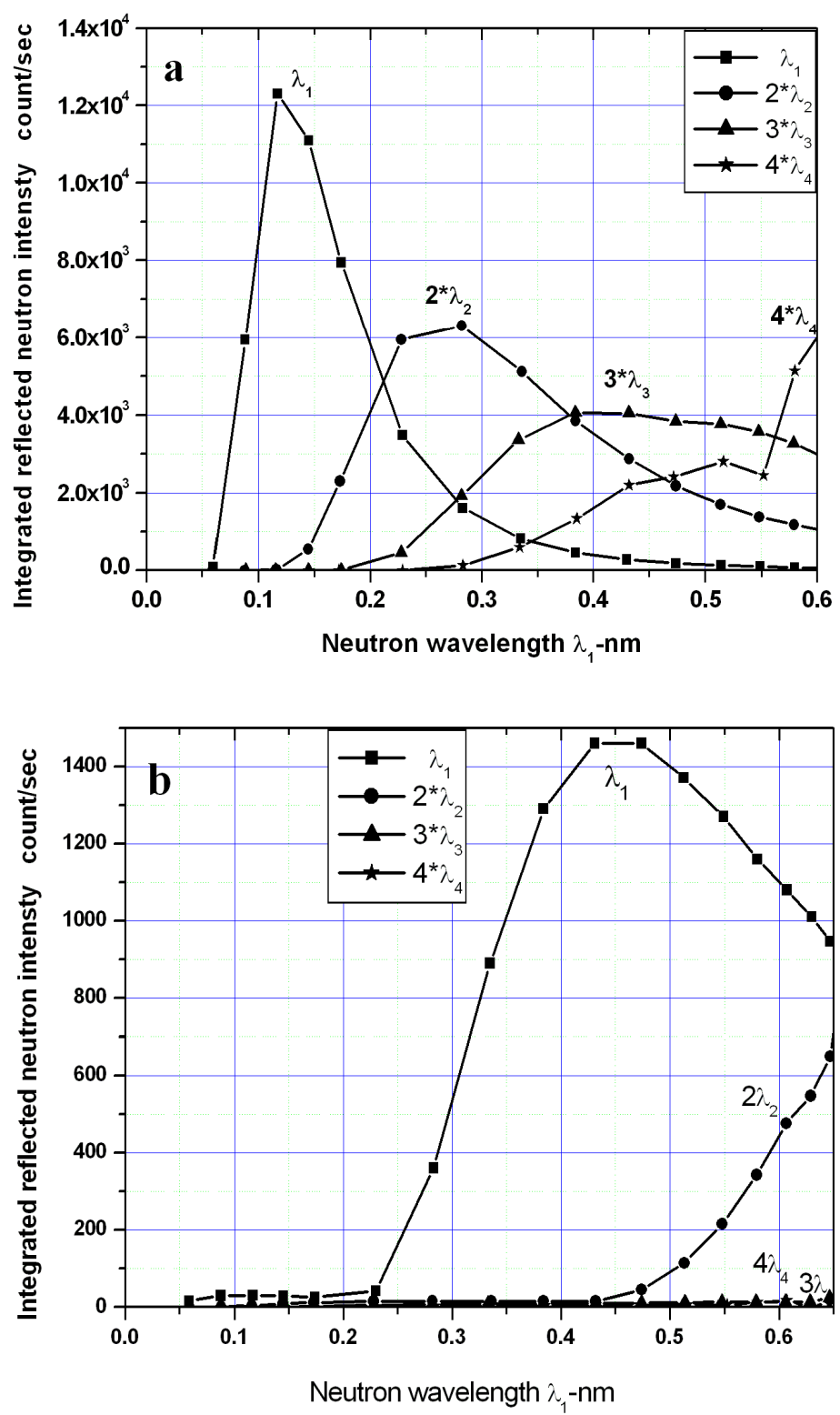

Figure 7. The integrated reflected monochromatic neutrons intensity versus wavelength $\lambda$

However, if a cold reactor flux is available, as shown from Figure 7b, the selected neutron wavelength band of the monochromatic cold beam is almost free from higher orders within the wavelength band from 0.25 to $0.5 \mathrm{~nm}$. Within such band, the contamination of second order is less than $5 \%$ and the higher orders are even less.

\section{Conclusion}

The developed Mono-PG code, used to calculate the reflectivity from PG crystal was found to be sufficient for determining its neutron monochromatic characteristics. It was shown that, the optimum FWHM on mosaic spread of PG when it used as a neutron monochromator is $0.3^{0}$, at $\Delta \lambda / \lambda \prec 3 \%$ and thickness from 2 to $5 \mathrm{~mm}$. However, when the thermal reactor flux is incident on PG crystal, the integrated reflectivity of $1^{\text {st }}$ order monochromatic neutrons with wavelengths $\lambda \geq 0.267 \mathrm{~nm}$ was even less than the $2^{\text {nd }}$, or the $3^{\text {rd }}$ orders ones. Therefore its use as thermal neutron monochromator is limited. While, when a cold reactor flux is incident, the reflected monochromatic neutrons from PG crystal is almost free from higher order contaminations. 


\section{References}

Adib, M., \& Fathalla, M. (2007). Computer package for graphite total cross-section calculations. 6th Nuclear and Particle Physics Conference, Luxor, Egypt, pp. 35-42.

Adib, M., Habib, N., \& Fathalla, M. (2006a). Neutron transmission through pyrolytic graphite crystals. Ann. Nucl. Energy, 33, 627-632. http://dx.doi.org/10.1016/j.anucene.2006.01.006

Adib, M., Habib, N., \& Fathalla, M. (2006b). Pyrolytic graphite as a selective neutron filter. Nucl. Phys. At. Energy, 2(18), 135-142.

Adib, M., Habib, N., Bashter, I., \& Saleh, A. (2011). Neutron transmission through pyroletic graphite crystal II. Journal Annals of Nuclear Energy, 38, 802-807. http://dx.doi.org/10.1016/j.anucene.2010.11.018

Arzi, E. (2001). Introduction to Neutron Powder Diffractometry. Wales: University College Cardiff Press Cardiff.

Bacon, G. E. (1975). Neutron Diffraction (3rd ed.). Oxford: Clarendon press.

Gurevich, I. I., \& Tarasov, L. V, (1968). Low-energy neutron Physics. North-Holland Publishing Company-Amsterdam.

Lovesey, S. W. (1987). Theory of Neutron Scattering from Condensed Matter, Vol. 1. Oxford: Oxford University Press

Mildner, D. F. R., Arif, M., \& Werner, S. A. (2001). Neutron transmission through pyrolytic graphite monochromator. J. Appl. Crystallogr, 34, 255-262. http://dx.doi.org/10.1107/S0021889801002254

Mitchell, P. (2006). Solid State Physics. Magic crystal balls Page 1 of 78 Taken from lectures at Manchester University. Retrieved from http://porlhews.tripod.com/sitebuildercontent/sitebuilderfiles/solidstatephysics.pdf

Mook, H. A., \& Hamilton, W. A. (2001). IN Advanced Optics for Neutron Scattering. Research, Annual Report, FY-ORNL.

Ohler, M., Baruchel, J., Moore, A. W., Ph. Galez, \& A. K. Freund. (1997). Direct observation of mosaic blocks in highly oriented pyrolytic graphite. Nucl. Instr. and Meth, B129, 257-260. 